\title{
Test-retest reliability and Practice Effect of the Leuven Perceptual Organisation Screening Test
}

4

5 Running head: L-POST reliability and practice effect

6

$7 \quad$ Xiaotong Ding ${ }^{1}$ and Kathleen Vancleef $* 1$

8

9

10

11

12

*Department of Experimental Psychology, University of Oxford, Oxford, OX2 6GG, United Kingdom

Email: kathleen.vancleef@psy.ox.ac.uk

ORCiD: 0000-0002-9943-9341

\section{Abstract:}

\section{Keywords:} Anna Watts Building, Radcliffe Observatory Quarter, Woodstock Road, University of

Visual diagnostic tests must have a high degree of consistency in its measurements (high reliability) to ensure accurate assessment of perceptual abilities. The current study assessed test-retest reliability and practice effects in Leuven Perceptual Organisation Screening Test (L-POST) in 144 healthy volunteers using correlation analysis, Bland-Altman analysis, and multi-level modelling. Results from three analyses converged and supported an adequate reliability of L-POST. Additionally, multi-level modelling demonstrated an absence of practice effect, suggesting that the L-POST is suitable for repeat administration.

Visual assessment, test-retest reliability, Bland-Altman analysis, practice effect 
28 Acknowledgements:

29 KV is supported by the Stroke Association [grant number TSA PDF 2017/03]. We would like

30 to thank Rudy Dekeerschieter, Christophe Bossens, and Johan Wagemans for programming

31 support and continued discussions about L-POST.

32 Total word count: 1500 
Visual perception is the extraction and interpretation of visual cues from the environment. It underlies our ability to acquire object knowledge and to interact effectively with others. However, it is easily affected by brain damage and neurological disorders (e.g., James, Culham, Humphrey, Milner, \& Goodale, 2003). Various tests have been developed to measure the perceptual abilities retained after brain damage, such as the Leuven Perceptual Organisation Screening Test (L-POST) (Torfs, Vancleef, Lafosse, Wagemans, \& deWit,2014). L-POST is a short online test consisting of 15 subtests each with five items. The subtests are designed to cover a range of mid-level processing, such as figure-background separation, texture/contour integration, object grouping, and figure completion.

To ensure accurate assessment of perceptual abilities, diagnostic tests must have high reliability - a high degree of consistency in its measurements (Cohen \& Swerdlik, 2018). The reliability of a study can be assessed in many ways. For example, test-retest reliability refers to the consistency of measurements taken at two separate times (Cohen \& Swerdlik, 2018). intervals around the observed correlations. Because samples vary randomly, it is plausible that the obtained correlation is much larger or smaller than the true population correlation. The smaller the sample size, the greater the likelihood of obtaining a spuriously small correlation coefficient. It is therefore uncertain if the low correlations reflect a genuine poor test-retest reliability or are extreme estimates due to their small sample size. Schonbrodt and 
Perugini (2013) simulated the effect of sample sizes on correlation estimates. They recommend a minimum sample size of 129 participants to find at least $95 \%$ of the sample correlations between values of 0.6 and 0.8 given a true population correlation of 0.7 .

Furthermore, correlation analysis examines the relatedness rather than agreement between two measurements. A high correlation coefficient can be obtained if all participants made a similar improvement in the retest (meaning that there is low agreement between test and retest). Notably, such a practice effect is not uncommon in neuropsychological assessment (Calamia, Markon, \& Tranel, 2012). Therefore, methods sensitive to the differences between measurements, such as the Bland-Altman analysis and multi-level models, are more preferable to evaluate test-retest reliability. Bland-Altman analysis reveals the discrepancy between two measurements by plotting their difference against their mean value for each corresponding pair of values (Bland \& Altman, 2010). Additionally, it is less affected by the range of values the variables take compared to correlation analysis (Karlijn, Kitty, Zoccali, \& Friedo, 2008). Bland-Altman analysis is therefore more appropriate in the case of L-POST subtests, where the range is limited to five items per subtests. Multi-level models add extra values by investigating the effect of additional variables like time between retests.

The current study aims to evaluate test-retest correlations of the L-POST in a sufficiently large sample. Additionally, we evaluate practice effects through Bland-Altman analyses and multi-level modelling.

\section{Method}

Our convenience sample consisted of 144 healthy volunteers (39 males, 105 females).

Participants' age ranged between 18.34 and 83.48 years (median $=23.28$, interquartile range $=19.92-35.62)$. The mean time spent in education was 14.36 years $(\mathrm{SD}=4.62)$. Participants' country of residence was: Belgium (58), Hungary (24), Italy (11), Turkey (10), 

reported normal (66) or corrected-to-normal (75) vision. Three participants reported to have an eye condition. None of the participants reported to have any neurological disorders. All procedures were approved by Commission for Medical Ethics of [location removed to enable double-blind review] (ML8800).

L-POST is a free online test for mid-level visual perception available at https://psytests.be/clinicians/ and is described in detail in Torfs et al. (2014). In 15 subtests of 5 items each, participants choose one stimulus out of three that best resembles the target stimulus shown at the top of the screen. Participants completed the L-POST at least twice and up to 18 times in their preferred language ( 8 options available) on their own device (large enough screen for simultaneous presentation of all stimuli) at their preferred time and location. Sessions where participants reported a high level of interruptions or technical issues were excluded from the analyses. We included 320 sessions of our 144 participants: 2 sessions from 132 participants, 3 sessions from 9 participants, 6 sessions from 1 participant, 10 session from 1 participant, and 13 sessions from 1 participant. The duration between sessions ranged between 4 minutes and 756 days, with a median of 16 days (interquartile range $=0-104$ days)

Reliability was evaluated by comparing performance on the first and second session for which data were available. Comparisons were made through test-retest correlations and Bland-Altman analysis. We calculated Pearson's correlation for the continuous variable 'total L-POST score' and polychoric correlations for the subtest scores. Permutation tests were used to calculate p-values. The Bland-Altman statistics were calculated using the 'blandr' package in R. The practice effect was explored using multi-level modelling using restricted 
maximum likelihood estimates with trial number and time between sessions as fixed effects and a random intercept to account for inter-individual differences in baseline performance.

\section{Results}

The Pearson's correlation of total scores of L-POST demonstrated adequate test-retest reliability $(\mathrm{r}=.70, \mathrm{p}<.001$, Figure $1 \mathrm{~A})$. Polychoric correlations of subtests ranged between 0.22 and 0.79 and were significantly different from zero for all but two subtests (Table 1).

Bland-Altman analyses showed a mean difference between test and retest total scores of $-0.26(\mathrm{SD}=5.34)$. The $95 \%$ confidence interval $(\mathrm{CI})$ for the mean difference was -1.14 to 0.62. The upper and lower limit of agreement (LoA) for total scores were 10.20 (95\% CI $[8.69,11.70])$ and $-10.72(95 \% \mathrm{CI}[-12.23,-9.22])$ respectively (Figure 1B). The mean difference for subtests ranged from -0.22 to 0.19 ; limits of agreement sit between -2.46 and 2.17 (Table 1).

$\underline{\text { Table 1. Test-retest correlation and Bland-Altman analysis for each subtest }}$

\begin{tabular}{|c|c|c|c|c|}
\hline & Vancleef et al. & Current study & & \\
\hline \multirow[t]{2}{*}{ Subtests } & \multirow{2}{*}{$\begin{array}{l}\text { Test-retest } \\
\text { reliability } \\
(\mathrm{n}=20)\end{array}$} & \multirow{2}{*}{$\begin{array}{l}\text { Test-retest } \\
\text { reliability } \\
(n=144)\end{array}$} & \multicolumn{2}{|c|}{ Bland-Altman analysis } \\
\hline & & & Mean difference & $\begin{array}{l}\text { Upper and lower } \\
\text { LoA }\end{array}$ \\
\hline $\begin{array}{l}\text { 1. Fine shape } \\
\text { discrimination }\end{array}$ & $.53(.07)$ & $.38(<.001)$ & $0.07[-0.09,0.23]$ & $1.93,-1.79$ \\
\hline $\begin{array}{l}\text { 2. Shape ratio } \\
\text { discrimination (Efron) }\end{array}$ & $.56(.16)$ & $.22(.17)$ & $0.03[-0.08,0.15]$ & $1.45,-1.38$ \\
\hline 3. Dot lattices & $.54(.04)$ & $.41(<.001)$ & $-0.06[-0.22,0.11]$ & $1.92,-2.03$ \\
\hline $\begin{array}{l}\text { 4. RFP fragmented } \\
\text { outline }\end{array}$ & $-.09(.82)$ & $.67(<.001)$ & $-0.05[-0.15,0.05]$ & $1.14,-1.24$ \\
\hline $\begin{array}{l}\text { 5. RFP contour } \\
\text { integration }\end{array}$ & $.46(.06)$ & $.59(<.001)$ & $0.03[-0.11,0.17]$ & $1.70,-1.64$ \\
\hline 6. RFP texture surface & $.67(.02)$ & $.61(<.001)$ & $-0.01[-0.14,0.13]$ & $1.57,-1.59$ \\
\hline $\begin{array}{l}\text { 7. Global motion } \\
\text { detection }\end{array}$ & $.94(<.01)$ & $.79(<.001)$ & $-0.09[-0.19,0.01]$ & $1.09,-1.27$ \\
\hline $\begin{array}{l}\text { 8. Kinetic object } \\
\text { segmentation }\end{array}$ & $.77(<.01)$ & $.77(<.001)$ & $0.02[-0.06,0.10]$ & $0.96,-0.92$ \\
\hline 9. Biological motion & $.51(.04)$ & $.64(<.001)$ & $-0.22[-0.40,-0.03]$ & $2.03,-2.46$ \\
\hline 10. Dot counting & $.56(.03)$ & $.55(<.001)$ & $0.19[0.03,0.35]$ & $2.11,-1.74$ \\
\hline
\end{tabular}




\begin{tabular}{|c|c|c|c|c|}
\hline $\begin{array}{l}\text { 11. Figure-ground } \\
\text { segmentation }\end{array}$ & $.60(<.01)$ & $.55(<.001)$ & $-0.01[-0.15,0.13]$ & $1.67,-1.69$ \\
\hline $\begin{array}{l}\text { 12. Embedded figure } \\
\text { detection }\end{array}$ & $-.03(.98)$ & $.53(<.001)$ & $-0.06[-0.24,0.13]$ & $2.17,-2.28$ \\
\hline $\begin{array}{l}\text { 13. Recognition of } \\
\text { missing part }\end{array}$ & $.50(.08)$ & $.45(<.001)$ & $-0.06[-0.22,0.10]$ & $1.82,-1.95$ \\
\hline 4-15. Object recognition & $.90(<.01)$ & $.28(.17)$ & $-0.06[-0.15,0.03]$ & $1.01,-1.13$ \\
\hline
\end{tabular}

122 Note. A bold value indicates a test-retest correlation that is significantly different from 0.

123 The $p$-values of the polychoric correlations are shown between brackets.

The $95 \%$ confidence intervals for mean differences in Bland-Altman analysis are shown between square brackets.

127 The multi-level model's intercept (baseline performance) was estimated at $64.70(t(173)=$ $109.85, p<.001)$. Within this model, the effect of trial number was not significant $(\beta=-$ $0.045, t(173)=-0.31, p=.760)$, neither was the effect of time between sessions $(\beta=0.004$, $t(173)=1.02, p=.308)$ or the interaction effect of trial number and time between sessions $(\beta$ $=-0.000, t(173)=-0.16, p=.870)$. Our multi-level model explained $70.60 \%$ of the variance

132 of total score (conditional $\mathrm{R}^{2}$ ). The variance explained by the fixed effects was only $0.26 \%$

133 (marginal $\mathrm{R}^{2}$ ). This discrepancy suggests high inter-individual variability in baseline scores 134 and little effect of repeated testing and the time between sessions. 

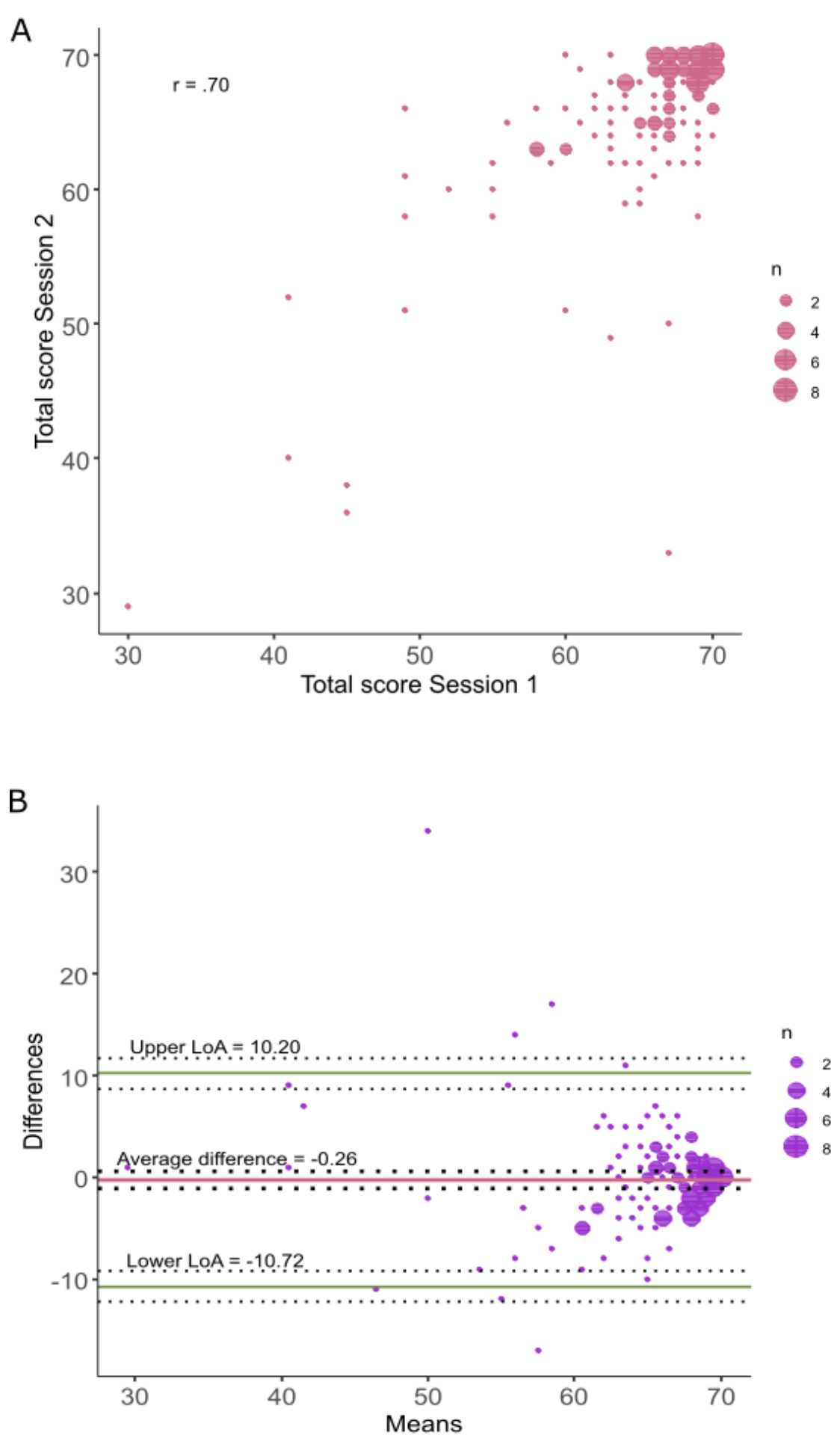

Figure 1. (A) A scatter plot depicting the correlation between total scores in Session 1 and Session 2. Pearson's correlation coefficient $r$ is presented at the top left corner of the graph $(r=.71)$. The size of the dots reflects the number of overlapping points, with bigger dots representing more measurements. (B) A Bland-Altman plot comparing the means and differences in total scores in Session 1 and Session 2. The central red line represents the average difference in total scores across the two sessions (average difference $=-0.26$ ). The limits of agreement are indicated by the green lines at the top and bottom of the graph (upper LoA $=10.20$, lower LoA $=$ -10.72). 95\% confidence intervals for the average difference, the upper LoA, and the lower LoA are shown as shaded areas and as black dotted lines above and below each of the three lines. The size of the dots reflects the number of overlapping points, with bigger dots representing more measurements. 


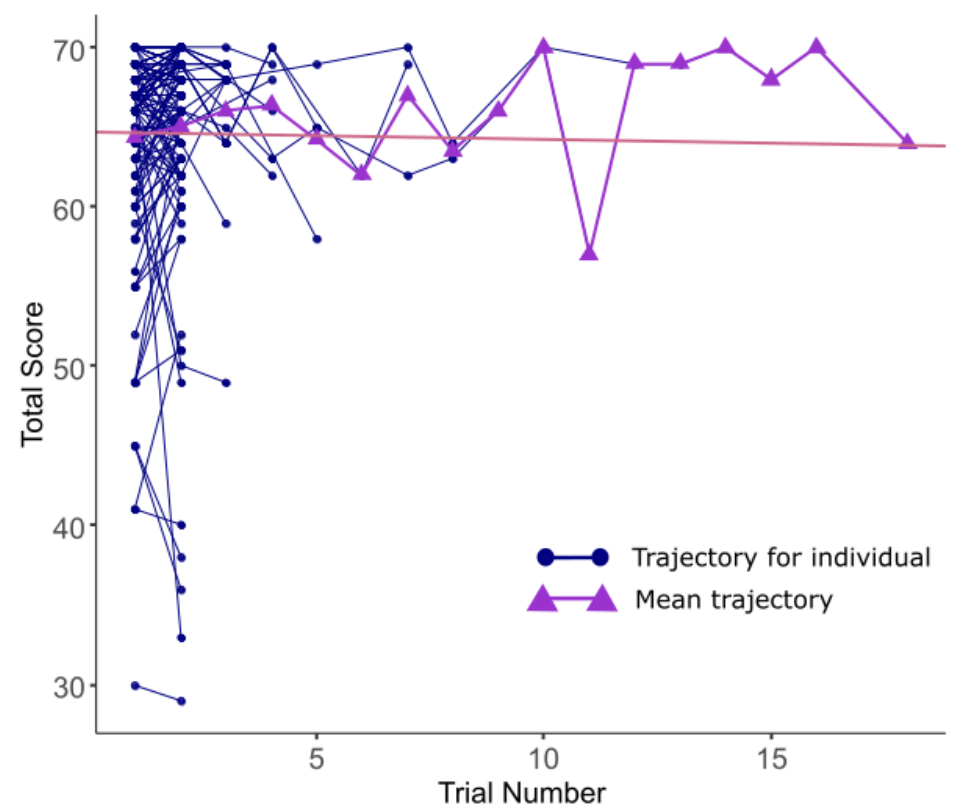

Figure 2. A spaghetti plot of total scores of 144 healthy volunteers at each L-POST trial. Sessions where participants reported a high level of interruptions or technical issues were excluded from the analyses. For instance, trial 17 of the one participant who completed 18 trials was excluded and therefore no data are shown for trial number 17. Individual trajectories for total score are indicated by blue dots with a thin line connecting the total scores of multiple trials for the same participant. The bold purple triangles and connecting line illustrates the trajectory of average score across trials. The red straight line represents the estimated regression line.

\section{Discussion}

The current study confirmed the results of Vancleef et al. (2015) in a group of 144 healthy volunteers. Test-retest correlation between the total scores of 0.70 suggests adequate reliability of L-POST. Our coefficient also fits the range of test-retest correlations found in other visual assessment (Brown et al. (2010). At the subtest level, we found test-retest correlations between 0.22 and 0.79 that were significantly different from zero in all except two of the subtests, further supporting adequate reliability of L-POST. In contrast to previous work, the current study employed Bland-Altman analyses and demonstrated good agreement 
between the measurements taken on two test occasions, supporting the conclusion from the correlation analyses. Furthermore, the results suggest that reliability of L-POST is stable across all levels of visual perceptual skills and that a change of at least 10 points in the total score represents a genuine difference. However, for each subtest limits of agreement fluctuated around \pm 2 , suggesting that any difference within two points may be due to measurement error. This can be problematic in the context of a five-point scale, where a twopoint difference represents a $40 \%$ change in score. Multi-level modelling confirmed the absence of a practice effect and showed that the time between sessions did not predict the total score. This suggests that the L-POST is suitable for repeat administration, even at short intervals.

Ceiling effects in L-POST scores might have negatively affected the correlations. However, our Bland-Altman analyses and multi-level modelling reached convergent results as the correlation analyses, supporting an adequate reliability of the L-POST. Future inclusion of patient populations would diversify the sample and minimise ceiling effects. Second, data were collected online with little control or knowledge of the test conditions. We compensated this lack of control by using strict inclusion criteria: only data with no reported technical issues or interruptions were included in the analyses.

In conclusion, this study demonstrated adequate reliability of L-POST and the absence of a practice effect.

Bland, J. M., \& Altman, D. G. (2010). Statistical methods for assessing agreement between two methods of clinical measurement. International Journal of Nursing Studies, 47(8), 931-936. doi:10.1016/j.ijnurstu.2009.10.001 
Brown, T., Sutton, E., Burgess, D., Elliott, S., Bourne, R., Wigg, S., . . Lalor, A. (2010). The reliability of three visual perception tests used to assess adults. Perceptual and Motor Skills, 111(1), 45-59. doi:10.2466/03.24.27.PMS.111.4.45-59

Calamia, M., Markon, K., \& Tranel, D. (2012). Scoring higher the second time around: Metaanalyses of practice effects in neuropsychological assessment. The Clinical Neuropsychologist, 26(4), 543-570. doi:10.1080/13854046.2012.680913

Cohen, R. J., \& Swerdlik, M. E. (2018). Psychological testing and assessment: An introduction to tests and measurement (9th ed.). New York: McGraw-Hill Education.

James, T. W., Culham, J., Humphrey, G. K., Milner, A. D., \& Goodale, M. A. (2003). Ventral occipital lesions impair object recognition but not object-directed grasping: An fMRI study. Brain, 126(11), 2463-2475. doi:10.1093/brain/awg248

Karlijn, J. V. S., Kitty, J. J., Zoccali, C., \& Friedo, W. D. (2008). Agreement between methods. Kidney International, 74(9), 1116. doi:10.1038/ki.2008.306

Schonbrodt, F., \& Perugini, M. (2013). At what sample size do correlations stabilize? Journal of Research in Personality, 47(5), 609-612. doi:10.1016/j.jrp.2013.05.009

Taylor, R. (1990). Interpretation of the correlation coefficient: A basic review. Journal of Diagnostic Medical Sonography, 6(1), 35-39. doi:10.1177/875647939000600106

Torfs, K., Vancleef, K., Lafosse, C., Wagemans, J., \& de-Wit, L. (2014). The Leuven Perceptual Organization Screening test (L-POST), an online test to assess mid-level visual perception. Behavior Research Methods, 46(2), 472-487. doi:10.3758/s13428013-0382-6 
209 Vancleef, K., Acke, E., Torfs, K., Demeyere, N., Lafosse, C., Humphreys, G., . . . De-Wit, L. 210 (2015). Reliability and validity of the Leuven Perceptual Organization Screening test (L211 POST. Journal of Neuropsychology, 9(2), 271-298. doi:10.1111/jnp.12050 